\title{
FATORES IMPACTANTES NA DECISÃO DE CERTIFICAÇÃO DA FIRMA: ALGUMAS CONSIDERAÇÕES PARA PEQUENAS E MÉDIAS EMPRESAS NO BRASIL
}

\section{FACTORS IMPACTING IN DECISION-FIRMA CERTIFICATION: SOME CONSIDERATIONS FOR SMALL AND MEDIUM BUSINESS IN BRAZIL}

\author{
FEITOSA ${ }^{1}$, Anny Kariny \\ Instituto Federal do Ceará - IFCE - Iguatu - CE, Brasil \\ CORREA, Márcio Veras \\ Centro de Pós-graduação em Economia - CAEN/UFC - Fortaleza - CE, Brasil
}

Recebido em: 29/08/2017; Aceito: 06/11/2017; Publicado: 01/12/2017

\begin{abstract}
Resumo: $\mathrm{O}$ presente artigo tem como objetivo identificar os fatores impactantes na decisão de certificação de pequenas e médias empresas brasileiras, utilizando uma amostra de 1.553 firmas de pequeno e médio porte, pesquisadas pelo Enterprise Surveys do Banco Mundial, durante o período de maio de 2008 a junho de 2009 no Brasil. Para tanto, estimou-se um modelo econométrico do tipo probit. Como resultados, tem-se que as variáveis que mais influenciam o aumento da probabilidade de certificação das pequenas e médias empresas brasileiras, para a amostra em estudo, são: site próprio $(13 \%)$, auditoria externa $(11,95 \%)$ e licença de importação $(10,08 \%)$.
\end{abstract}

Palavras Chaves: Certificação, Normas ISO, Pequenas e Médias Empresas.

Abstract: This article aims to identify the factors impacting on the certification decision of small and medium companies in Brazil, using a sample of 1,553 firms, small and medium, surveyed by the World Bank Enterprise Surveys, during the period from May 2008 to June 2009 in Brazil. To do so, we estimated a probit econometric model type. As a result, we have that the variables that most influence the increased likelihood of certification of Brazilian small and medium enterprises, for the sample under study are: own website (13\%), external audit (11.95\%) and import license $(10.08 \%)$.

Keyword: Certification, ISO Standards, Small and Medium Enterprises.

\footnotetext{
${ }^{1}$ Mestre em Economia, UFC. Docente no Instituto Federal do Ceará - IFCE. Contato: anny.feitosa@ifce.edu.br
} 


\section{INTRODUÇÃO}

Atualmente, as certificações de produto e processo, relacionadas à qualidade e/ou gestão ambiental e social, tornaram-se requisitos mínimos indispensáveis para que as empresas possam ter acesso ao mercado internacional. Neste sentido, a gestão da qualidade no ambiente das pequenas e médias empresas pode ser entendida como uma forma de permitir sua sobrevivência no mercado econômico globalizado.

Em todo o mundo, a referência para a certificação de processos é a Organização Internacional para a Normalização - ISO, que surgiu a partir da confederação internacional de órgãos nacionais de normalização (ONNs) e estabelece práticas internacionalmente aceitas, que servem como base para o funcionamento do comércio internacional, permitindo às empresas o benefício de serem mais eficientes, tendo em vista as mesmas normas serem aplicadas em todos os mercados e setores em que atuam. (SEBRAE e ABNT, s.d.).

As normas mais comumente adotadas em todo o mundo são a série da família ISO 9000, que envolve elementos de conceituação da qualidade e mecanismos relativos a seu planejamento, gestão, implantação e avaliação (ISO, 2009a); e, a séria ISO 14000, que aborda assuntos, tais como: Sistemas de Gestão Ambiental - SGA, Auditorias Ambientais, Rotulagem Ambiental, Avaliação do Ciclo de Vida e Comunicação Ambiental (ISO, 2009b). Ambas servem como referência para a certificação internacional na presente pesquisa.

A família ISO 9000 representa um consenso internacional sobre boas práticas de gestão, estabelecendo um "padrão genérico de gestão", enquanto a ISO 14000 pode beneficiar as empresas através da redução dos custos de energia e melhorando sua imagem com o público em geral e outras partes interessadas (HUDSON E ORVISKA, 2013).
Parafraseando Szyszka (2001), é possível citar motivos para a busca da certificação, que visam melhorar a capacidade de atendimento, manter ou aumentar o mercado de atuação, melhorar a qualidade e produtividade da empresa, atender às necessidades de clientes, ganhar vantagens de marketing $e$ melhorar a eficiência e o desempenho competitivo da empresa.

Contudo, estudos sobre o desempenho competitivo das micro, pequenas e médias empresas (MPMEs) indicam que existem limitações a este desempenho que são comuns às empresas de países desenvolvidos e em desenvolvimento, tais como máquinas obsoletas, administração inadequada e dificuldades de comercialização de seus produtos em novos mercados (LEVISTKY 1996), somam-se a isso condições de logística inadequadas e a estrutura tributária complexa no caso brasileiro.

Não obstante, é evidente a relevância dos pequenos e médios negócios e a sua contribuição no crescimento econômico, no desenvolvimento social e no processo de distribuição de renda no país. Ademais, as unidades produtivas de micro e pequeno porte são apontadas como as responsáveis pela maioria dos postos de trabalho gerados, avançando, a cada ano, na oferta de emprego e na ocupação de novos segmentos de mercado devido às menores necessidades de capital (KASSAI apud MOTTA, 2000).

Porém, quanto o assunto é certificação, as pequenas e médias empresas brasileiras apresentam baixa participação. Sabe-se, ainda, que as MPEs têm dificuldade de identificar e entender como as normas técnicas afetam as suas atividades. Frequentemente, não sabem que existem normas que se aplicam aos seus produtos (ou serviços) e muito menos que outras normas as afetam, seja por que se aplicam aos seus processos, seja porque se aplicam aos seus fornecedores ou aos seus clientes, refletindo-se naturalmente nas suas próprias atividades. Hudson e Orviska (2013) ao testarem os fatores que influenciavam a probabilidade 
de certificação de empresas na Ásia e no leste Europeu apontaram que as empresas menores enfrentam problemas no acesso ao conhecimento necessário para implementar os procedimentos pertinentes.

O problema é agravado quando se considera a possibilidade de que iniciativas de normalização, ao nível regional e ao nível internacional, possam afetar o seu negócio, o que é cada vez mais comum devido à crescente integração comercial na economia globalizada. Por essa razão, o desafio da inserção das MPEs no processo de normalização e na utilização de normas técnicas tem sido uma preocupação constante dos organismos de normalização nacionais e das entidades representativas dos micro e pequenos empresários no mundo todo (SEBRAE e ABNT, s.d.)

Partindo do exposto, este artigo tem o objetivo de identificar os fatores impactantes na decisão de certificação internacional de pequenas e médias empresas brasileiras, considerando características como a localização, o tamanho da firma, a ocorrência de auditoria externa, a existência de site próprio, o uso de tecnologia estrangeira e se a empresa recorreu à licença de importação.

\section{CERTIFICAÇÃO E NORMAS ISO}

\section{Certificação}

Certificação é a confirmação de que um produto, processo ou serviço satisfaz os padrões de uma determinada norma. Consiste em um procedimento mediante o qual uma terceira parte - o certificador assegura que um produto possui requisitos previamente estabelecidos (De CARLOS, 2007; ICONTEC, 2008 apud ACOSTA et.al , 2009).

Por sua vez, a norma ou normalização consiste na especificação de critérios ou requisitos a serem aplicados na escolha e classificação de materiais, na fabricação dos produtos e no fornecimento de serviços, resultante de acordos de consenso alcançados entre os sujeitos envolvidos em um determinado setor, sejam fornecedores, usuários e demais parceiros, com o objetivo de facilitar o comércio, intercâmbio e a transferência de tecnologia (SZYSZKA, 2001)

Segundo dados do Instituto Nacional de Metrologia, Qualidade e Tecnologia - INMETRO, a certificação, no âmbito do Sistema Brasileiro de Certificação (SBC), pode ser compulsória ou voluntária: estando a compulsória prioritariamente vinculada à segurança, ao interesse do país e do cidadão, abrangendo questões relativas aos animais, vegetais, proteção da saúde, do meio ambiente e temas correlatos; enquanto a voluntária é de decisão exclusiva do solicitante e tem como objetivo garantir a conformidade de processos, produtos e serviços com base nas normas brasileiras, regionais ou internacionais, dentro do conceito de níveis de normalização.

Especialmente a certificação voluntária surge como resposta ao nível crescente de exigência dos consumidores por produtos de mais alta qualidade, incluindo componentes ou matérias-primas, processos de transformação e distribuição (ICONTEC, 2008 apud ACOSTA et.al , 2009).

De acordo com Hudson e Orviska (2013), a adoção da certificação, por parte da firma, pode ser necessária para a entrada em determinados mercados, sendo útil ainda para melhorar as relações da empresa com a comunidade local, clientes, acionistas, trabalhadores e outros parceiros.

Deste modo, a certificação é um sistema de reforço dos atributos do produto e requer a existência de um padrão e uma penalização em caso de descumprimento das normas, com a finalidade de evitar fraudes, por meio de sistemas de controle mais rigorosos, gerando, assim, confiança no consumidor (CRUZ et al., 2004; COMPÉS, 2002 apud ACOSTA et.al , 2009). 
Segundo Szyszka (2001), pesquisas realizadas com organizações apontam como principais motivos para a busca da certificação: melhorar a capacidade de atendimento; manter ou aumentar o market share; melhorar a qualidade e produtividade; atender às exigências de clientes; ganhar vantagens de marketing; $e$ melhorar a eficiência.

A literatura se remete, ainda, ao fato de normas não harmonizadas para tecnologias similares em diferentes países ou regiões contribuírem para as chamadas barreiras técnicas para o comércio. Por esta razão, há a necessidade de que se proceda a normalização internacional com vistas a obter, entre outros fatores, padrões globais para tecnologias emergentes e a necessidade de uma infraestrutura em normalização para melhoria da produtividade, da competitividade e da capacidade de exportação dos países em desenvolvimento (SZYSZKA, 2001).

Considerando a relevância da certificação internacional, Hudson e Orviska (2013) analisaram os fatores que impactam na decisão das empresas em recorrer à referida certificação. E, concluíram que a probabilidade de obter certificação internacional aumenta com o tamanho da empresa, sendo maior em empresas situadas em cidades grandes, com predominância no segmento industrial.

Por sua vez, Gebreeyesus (2014) demonstrou que o tamanho da empresa, idade e propriedade estrangeira estão associados positivamente com a decisão de adoção de normas de certificação internacionais, que implica que as empresas maiores, mais antigas e de propriedade estrangeiras são mais propensas a adotar tais normas.

\section{Normas ISO}

A Organização Internacional para a Normalização - ISO, fundada em 1946, é uma organização não governamental, que surgiu a partir da confederação internacional de órgãos nacionais de normalização (ONNs) de todo o mundo. Seu principal objetivo é a publicação de documentos - Normas ISO para o estabelecimento de práticas internacionalmente aceitas, que servem como base para o funcionamento do comércio internacional, permitindo às empresas 0 benefício de serem mais eficientes, tendo em vista as mesmas normas serem aplicadas em todos os mercados e setores em que atuam. (SEBRAE e ABNT, s.d.).

A seguir, estão elencadas as normas ISO 9000 e ISO 14000, que servem como referência para a certificação internacional na presente pesquisa.

\section{ISO 9000: Gestão da Qualidade}

O Sistema ISO 9000, criado em 1987, valese do princípio de Gestão da Qualidade, segundo o qual, "garantindo-se a qualidade dos processos, garante-se a qualidade de produtos e serviços deles procedentes" (PALADINI, 2002). O sistema envolve elementos de conceituação da qualidade e mecanismos relativos a seu planejamento, gestão, implantação e avaliação.

Segundo Hudson e Orviska (2013), a família ISO 9000 representa um consenso internacional sobre boas práticas de gestão, estabelecendo um "padrão genérico de gestão".

Detalhando as normas da série 9000, segundo Paladini (2002), temos:

ISO 9000: trata de conceitos básicos sobre qualidade e lista cinco conceitos fundamentais: política da qualidade; administração da qualidade; sistema da qualidade; controle da qualidade; e, garantia da qualidade.

ISO 9001: apresenta as normas contratuais para sistemas de garantia da qualidade em nível de projeto, desenvolvimento, produção, instalação e assistência técnica.

ISO 9002: destina-se fundamentalmente à prevenção e detecção de qualquer ausência de conformação durante o processo produtivo e de instalação. 
ISO 9003: trata do modelo para a garantia da qualidade em inspeções finais e testes a serem realizados na conformação do produto.

ISO 9004: fornece diretrizes gerais para o desenvolvimento e a implementação dos sistemas da qualidade, tais como objetivos organizacionais, responsabilidades da administração, princípio dos sistemas da qualidade, qualidade em marketing, qualidade e controle na produção, entre outros aspectos.

Posteriormente, surge a ISO 19011, que abrange a área de auditoria de sistemas de gestão da qualidade e sistemas de gestão ambiental, fornecendo orientação sobre programas de auditoria, interna ou externa, oferecendo uma visão geral de como um programa de auditoria deve funcionar (ISO, 2009a).

Para a obtenção desta certificação, é possível identificar como requisitos: Foco no cliente; Objetivos da qualidade; Planejamento da qualidade; Comunicação interna; Provisão de recursos; Outros recursos; Planejamento da produção; Identificação dos requisitos do produto; Revisão dos requisitos do produto; Comunicação com o cliente; Validação de processos; Planejamento; Satisfação do cliente; Medição e monitoramento dos processos; e, Planejamento para a melhoria contínua; Responsabilidade da administração; Gerenciamento de recursos; Produção; Medição, análise e melhoria (ISO, 2009a).

Conforme Oliveira (2001), para que se proceda com a devida certificação, em atendimento aos requisitos descritos na norma, é necessário o cumprimento de etapas fundamentais, que são: Comprometimento da alta administração; Seleção e designação formal de um coordenador; Formação do Comitê de coordenação; Treinamento; Elaboração e divulgação da política da qualidade; Palestra sobre qualidade para todos os funcionários;

Divulgação constante do assunto qualidade; Estudo de cada um dos requisitos da norma e realização do diagnóstico da organização em relação ao requisito;
Plano de trabalho para implantação de cada requisito; Formação de grupos de trabalho com a participação dos funcionários para elaborar as instruções de trabalho; Elaboração do manual da qualidade; Treinamento dos funcionários na documentação da qualidade; Formação dos auditores internos da qualidade; Realização das auditorias internas; Implantação das ações corretivas para as não conformidades; Seleção da entidade certificadora; Realização da pré-auditoria; e, Realização da auditoria de certificação.

Ressalta-se que, dentre os requisitos estabelecidos para a certificação da ISO, figuram especialmente a necessidade de treinamento e a realização de auditorias, o que oferece subsídios para acreditar que as empresas que investem na capacitação de seus colaboradores e na prática de auditorias possuem uma maior probabilidade de obter a certificação.

\section{ISO 14000: Gestão Ambiental}

A família de normas ISO 14000, foi criada em 1991 dada a necessidade de desenvolvimento de normas internacionais relacionadas à área da gestão ambiental, indicando às empresas o que devem fazer para minimizar os impactos ambientais de suas atividades, oportunizando, assim, a melhoria contínua de seu desempenho ambiental. O referido sistema aborda assuntos, tais como: Sistemas de Gestão Ambiental (SGA), Auditorias Ambientais, Rotulagem Ambiental, Avaliação do Ciclo de Vida e Comunicação Ambiental (NICOLELLA et. al., 2004).

De acordo com dados encontrados no portal ISO, na internet, a família ISO 14000 contempla as seguintes normas (ISO, 2009b):

ISO 14001: trata dos principais requisitos para as empresas identificarem, controlarem e monitorarem seus aspectos ambientais, através de um sistema de gestão ambiental. 
ISO 14004: complementa a ISO 14001 provendo diretrizes adicionais para implantação de um sistema de gestão ambiental.

ISO 14031: guia para avaliação de desempenho ambiental.

ISO 14020: conjunto de normas que tratam de selos ambientais.

ISO 14040: conjunto de normas para conduzir análises de ciclo de vida de produtos e serviços.

ISO 14064: contabilização e verificação de emissões de gases de efeito estufa para suportar projetos de redução de emissões.

ISO 14065: complementa a ISO 14064 especificando os requisitos para certificar ou reconhecer instituições que farão validação ou verificação da norma ISO 14064 ou outras especificações importantes.

ISO 14063: trata de comunicação ambiental por parte das empresas.

ISO 14045: requisitos para análises de ecoeficiência.

ISO 14051: norma para MFCA - Material Flow Cost Accounting, ou em tradução literal, contabilidade de custos dos fluxos de materiais, uma ferramenta de gerenciamento que busca maximizar a utilização de recursos, principalmente em manufatura e processos de distribuição.

ISO 14067: norma para pegada de carbono em produtos, tratando de requisitos para contabilização e comunicação de emissões de gases de efeito estufa associados a produtos.

ISO 14069: guia para as empresas calcularem a pegada de carbono em seus produtos, serviços e cadeia de fornecimento.

ISO 14005: guia para a implementação em fases de um sistema de gestão ambiental para facilitar sua adoção por pequenas e médias empresas.

ISO 14006: norma para "ecodesign".
ISO 14033: diretrizes e exemplos para compilar e comunicar informações ambientais quantitativas.

ISO 14066: requisitos para as empresas que farão a validação e a verificação de emissões de gases de efeito estufa.

Hemenway and Hale (1995) apud Hudson e Orviska (2013), argumentam que a ISO 14000 pode beneficiar as empresas através da redução dos custos de energia e melhorando sua imagem com o público em geral e outras partes interessadas.

\section{METODOLOGIA}

A presente pesquisa foi viabilizada por meio da utilização da base de dados do Banco Mundial (Enterprises Surveys), que entrevistou empresas brasileiras, no período de maio de 2008 a junho de 2009, e consiste em um levantamento de informações de empresas que representam o setor privado da economia. Para o Brasil, a pesquisa considerou 1802 empresas registradas, que representam o universo do presente estudo, sendo 815 pequenas empresas, 738 médias empresas e 249 grandes empresas.

Entretanto, para a realização do presente estudo, a amostra da pesquisa levou em consideração as empresas brasileiras, de pequeno e médio porte, constantes na base da Enterprises Survey, que totalizam 1.553 empresas investigadas. Apesar da literatura na área acreditar haver uma relação positiva entre o tamanho da firma e a probabilidade de certificação, optou-se por excluir da presente análise as empresas de grande porte, na tentativa de observar como se comportam as pequenas e médias empresas brasileiras no processo de certificação de seus produtos, processos ou serviços, dada sua importância no contexto de crescimento econômico, geração de postos de trabalhos e menor necessidade de capital.

Para tanto, fez-se uso da classificação abordada pelo Banco Mundial, no âmbito da pesquisa 
Enterprises Survey, que considera pequenas empresas as detentoras de 5 a 19 empregados, médias empresas as que possuem de 20 a 99 empregados e grandes empresas aquelas com 100 ou mais empregados (BANCO MUNDIAL, n.d).

Seguindo a técnica proposta por Hudson e Orviska (2013), para investigar a probabilidade da decisão de certificação internacional de pequenas e médias empresas brasileiras, a estratégia metodológica constituiu-se em estimar um modelo probit, que é um caso particular de modelo de resposta no qual a variável dependente é binária e assume somente dois valores, geralmente 0 para a não-ocorrência do fenômeno e 1 para a ocorrência (PINO, 2007).

As variáveis consideradas para a análise foram selecionadas de acordo com a disponibilidade de informações na base de dados e parecem fornecer um quadro razoável de características e de variáveis relacionadas com o processo de certificação.

\section{Variável dependente limitada:}

Certificação (certificacao) - variável binária, que determina se a empresa tem Certificação Internacional de Qualidade (ISO 9000/ ISO 14000), atribuindo-se valor 1 (um) em caso afirmativo e 0(zero) caso contrário.

\section{Variáveis independentes:}

Tamanho da firma (tamanho) - é uma medida representada pela quantidade de trabalhadores permanentes e temporários que a empresa possui, considerando-se pequena (5 a 19 empregados) e média (20 a 99 empregados). Adotou-se 1(um) para média empresa e 0 (zero) para pequena. Como resultado, acredita-se que há uma relação positiva entre o tamanho da firma e a probabilidade de certificação, isto porque, dentre outros motivos, as

empresas menores enfrentam problemas no acesso ao conhecimento necessário para implementar os procedimentos pertinentes ao processo de certificação (GROLLEAU, MZOUGHI \& PEKOVIC, 2007 apud HUDSON E ORVISKA, 2013).

Localização (local) - A pesquisa relacionou empresas situadas nos maiores centros de produção do país. Entretanto, foi possível identificar que há empresas na capital e outras nas demais cidades nos Estados da Federação. Assim, utilizou-se 1(um) para Capital e 0(zero) para Interior. Como resultado, esperase encontrar maior probabilidade de empresas com certificação em capitais, onde há mais acesso aos agentes certificadores, bem como os efeitos da certificação podem ser obervados mais prontamente (HUDSON E ORVISKA, 2013).

Auditoria Externa (audiexterna) - Esta variável indica se a empresa tem suas demonstrações financeiras anuais verificadas e certificadas por um auditor externo. Considerou-se 1(um) em caso afirmativo e 0(zero) caso contrário. Espera-se encontrar uma relação positiva entre a existência de auditoria externa e a probabilidade de certificação.

Tecnologia Estrangeira (tecestrang) - Se a empresa usa tecnologia de uma empresa de propriedade estrangeira, com exclusão de software de escritório, sendo 1(um) em caso afirmativo e 0(zero) caso contrário. Considera-se que o uso de tecnologia estrangeira corrobora com a probabilidade de certificação.

Site Próprio (siteproprio) - Se a empresa utiliza site próprio para as atividades relacionadas ao negócio, ou seja, vendas, promoção de produtos, etc. Atribuindo-se 1(um) em caso afirmativo e 0(zero) caso contrário. Espera-se apontar uma relação positiva entre o uso de site próprio e a probabilidade de certificação, sendo o site um indicativo de inovação.

Licença de importação (licenseimport) Identifica se a empresa, nos últimos dois anos, apresentou pedido para obter licença de importação. Sendo 1 (um) em caso afirmativo e 0(zero) caso 
contrário. Admite-se uma relação positiva entre o pedido ou obtenção da licença de importação e a probabilidade de certificação.

\section{ANÁLISE DESCRITIVA DA PESQUISA}

De acordo com os dados utilizados na pesquisa, foi possível traçar o diagnóstico do perfil das pequenas e médias empresas brasileiras, participantes da pesquisa. Conforme dados da Tabela 1, que segue abaixo, há expressiva concentração das empresas participantes da pesquisa nas regiões Sudeste (661 empresas) e Sul (447 empresas) do país, que juntam perfazem o total de $71,67 \%$. Observa-se a participação de 234 empresas localizadas no Nordeste do Brasil, equivalente a 17,07\%; e um pequeno número de empresas localizadas na região Centro-Oeste (165) que somam $10,62 \%$, bem como na região Norte do país, com 46 empresas e 2,96\%.

TABELA 1 - Pequenas e Médias

\begin{tabular}{cccc}
\multicolumn{4}{c}{ Empresas por Região } \\
\hline Região & $\begin{array}{c}\text { Média } \\
\text { Empre } \\
\text { Sa }\end{array}$ & $\begin{array}{c}\text { Pequena } \\
\text { Empres } \\
\text { a }\end{array}$ & Total \\
\hline Sudeste & 335 & 326 & 661 \\
Sul & 196 & 251 & 447 \\
Nordest & 124 & 110 & 234 \\
e & & & \\
Centro- \\
oeste
\end{tabular}

Fonte: Elaboração Própria, 2014.

Com relação ao quesito certificação, 1525 empresas informaram serem ou não certificadas, conforme pode ser visto na Tabela 2, abaixo. Como resultado, tem-se que as empresas respondentes não possuem certificação em $85,44 \%$ dos casos, o que equivale a 1303 empresas, enquanto apenas 222 possuem (14,56\%). Além disso, é possível identificar que, dentre as certificadas, há predominância de médias empresas, com 153 empresas, contra 69 pequenas.

\begin{tabular}{cccc}
$\begin{array}{c}\text { TABELA } \\
\text { Empresas Certificadas }\end{array}$ & - Pequenas e Médias \\
\hline $\begin{array}{c}\text { Certific } \\
\text { ação }\end{array}$ & $\begin{array}{c}\text { Pequena } \\
\text { Empresa }\end{array}$ & $\begin{array}{c}\text { Média } \\
\text { Empres } \\
\text { a }\end{array}$ & Total \\
\hline Não & 736 & 567 & 1303 \\
Sim & 69 & 153 & 222 \\
Total & 805 & 720 & 1525
\end{tabular}

Fonte: Elaboração própria, 2014

Tal afirmativa encontra fundamento no trabalho de Grolleau, Mzoughi \& Pekovic (2007) apud Hudson \& Orviska (2013), ao afirmarem que empresas menores enfrentam problemas no acesso ao conhecimento necessário para implementar os procedimentos pertinentes ao processo de certificação.

A seguir, na Tabela 3 , estão relacionadas às estatísticas descritivas da amostra.

TABELA 3 - Estatísticas Descritivas da Amostra

\begin{tabular}{|c|c|c|c|c|c|}
\hline Variável & $\begin{array}{c}\text { Ob } \\
\mathbf{s}^{*}\end{array}$ & $\begin{array}{c}\text { Méd } \\
\text { ia }\end{array}$ & $\begin{array}{c}\text { Desvio } \\
\text { Padrã } \\
\text { o }\end{array}$ & Mínimo & $\begin{array}{c}\text { Máx } \\
\text { imo }\end{array}$ \\
\hline $\begin{array}{l}\text { Certificaçã } \\
\text { o }\end{array}$ & $\begin{array}{c}152 \\
5\end{array}$ & $\begin{array}{c}0,14 \\
5573 \\
8\end{array}$ & $\begin{array}{c}.35279 \\
41\end{array}$ & 0 & 1 \\
\hline $\begin{array}{l}\text { Tamanho } \\
\text { da empresa }\end{array}$ & $\begin{array}{l}15 \\
53\end{array}$ & $\begin{array}{c}0,47 \\
5209 \\
3\end{array}$ & $\begin{array}{c}0.4995 \\
459\end{array}$ & 0 & 1 \\
\hline $\begin{array}{l}\text { Localizaçã } \\
\text { o }\end{array}$ & $\begin{array}{l}15 \\
50\end{array}$ & $\begin{array}{c}0,56 \\
1290 \\
3\end{array}$ & $\begin{array}{c}0,4963 \\
894\end{array}$ & 0 & 1 \\
\hline $\begin{array}{l}\text { Auditoria } \\
\text { Externa }\end{array}$ & $\begin{array}{l}15 \\
36\end{array}$ & $\begin{array}{c}0,19 \\
3359 \\
4\end{array}$ & $\begin{array}{c}0,3950 \\
609\end{array}$ & 0 & 1 \\
\hline $\begin{array}{l}\text { Site } \\
\text { Próprio }\end{array}$ & $\begin{array}{l}15 \\
48\end{array}$ & $\begin{array}{c}0,59 \\
4315 \\
2\end{array}$ & $\begin{array}{c}0,4911 \\
827\end{array}$ & 0 & 1 \\
\hline $\begin{array}{l}\text { Tecnologia } \\
\text { Estrangeira }\end{array}$ & $\begin{array}{l}11 \\
08\end{array}$ & $\begin{array}{c}0,12 \\
0036 \\
1\end{array}$ & $\begin{array}{c}0,3251 \\
505\end{array}$ & 0 & 1 \\
\hline $\begin{array}{l}\text { Licença de } \\
\text { Importação }\end{array}$ & $\begin{array}{l}15 \\
51\end{array}$ & $\begin{array}{c}0,14 \\
8936 \\
2\end{array}$ & $\begin{array}{c}0,3561 \\
404\end{array}$ & 0 & 1 \\
\hline
\end{tabular}


Fonte: Elaboração própria, 2014. *O número de observações é diverso, conforme a disponibilidade da informação na base de dados consultada para cada variável. A amostra total compõe-se de 1553 empresas.

A Tabela 3 apresenta a relação de variáveis, o número de observações, bem como a média, o desvio padrão, mínimo e máximo relacionados com as respostas das empresas. Ressalta-se que as variáveis são predominantemente binárias, tendo suas respostas entre 0 e 1 .

Com relação à média, pode-se afirmar conforme segue:

Dentre as empresas consultadas, a certificação está presente em 14,56\% dos casos, o que confirma a informação constante na Tabela 2, de que $85,44 \%$ das empresas da amostra não possuem certificação.

A respeito do tamanho da empresa, 47,52\% das empresas respondentes são médias empresas e $59,43 \%$ se constituem de pequenas empresas; Cerca de $56,13 \%$ das empresas consultadas na pesquisa estão localizadas em capitais do Brasil, enquanto 43,87\% estão situadas nas demais cidades; Sobre a ocorrência de auditoria externa, $19,34 \%$ das empresas respondentes alegam se submeterem a auditorias em seus processos, enquanto $80,66 \%$ não o fazem.

Quando perguntadas sobre o fato de possuírem site próprio, 59,43\% responderam positivamente, enquanto $40,57 \%$ empresas admitiram não possuir; Em apenas $12 \%$ dos casos, as empresas fazem uso de tecnologia estrangeira, resultando em $88 \%$ de empresas que não dispõe; Das empresas consultadas na pesquisa, $14,89 \%$ dos casos são detentoras de licença para importação, o que não ocorre em $85,11 \%$.

\section{RESULTADOS}

Os resultados apresentados a seguir referemse à estimação do modelo econométrico utilizado para determinar os fatores impactantes na decisão de certificação internacional de pequenas e médias empresas brasileiras, conforme segue na Tabela 4 .

TABELA 4 - Resultado da Regressão

CERTIFICACAO

\begin{tabular}{|c|c|c|c|}
\hline Variáveis & $\begin{array}{c}\text { P- } \\
\text { valor }\end{array}$ & Coef & EM\% \\
\hline tamanho & $\begin{array}{c}0,004 \\
*\end{array}$ & $0,3111(2,84)$ & 5,74 \\
\hline local & $\begin{array}{c}0,023 \\
* *\end{array}$ & $\begin{array}{c}-0,2381(- \\
2,27)\end{array}$ & $-4,39$ \\
\hline audiexterna & $\begin{array}{c}0,000 \\
*\end{array}$ & $0,5349(4,44)$ & 11,95 \\
\hline siteproprio & $\begin{array}{c}0,000 \\
*\end{array}$ & $0,7551(6,00)$ & 13,00 \\
\hline tecestrang & $\begin{array}{c}0,057 \\
* * *\end{array}$ & $0,2733(1,90)$ & 5,68 \\
\hline licenseimport & $\begin{array}{c}0,000 \\
*\end{array}$ & $0,4557(3,54)$ & 10,08 \\
\hline Observações & & 1066 & \\
\hline Log Likelihood & -369 & & \\
\hline$X^{2}$ & & 147.67 & \\
\hline Prob > chi 2 & & 0.0000 & \\
\hline Pseudo $R^{2}$ & & 0.1664 & \\
\hline \%correta & & $85.55 \%$ & \\
\hline
\end{tabular}

Fonte: elaboração própria, 2014. Notas: (.) indica estatísticas $t ; \quad * / * * / * * *$ representam o nível de significância a $1 \%, 5 \%$ e $10 \%$, respectivamente; $X^{2}$ indica o teste da razão de verossimilhança; \%correta representa em termos percentuais que o modelo previu corretamente as observações.

Conforme se observa na Tabela 4, estimou-se a probabilidade da decisão de certificação da firma ser afetada, positiva ou negativamente, pelas variáveis: tamanho da firma, localização, auditoria externa, site próprio, tecnologia estrangeira e licença de importação.

Como resultado, tem-se que a modelagem com tais variáveis rejeita a hipótese nula de que nenhuma das variáveis exógenas exerce influência sobre a variável endógena, baseando-se no teste da razão de verossimilhança $\left(X^{2}\right)$ encontrado de 147,67 ; no nível de significância do modelo, que, atribuído por um valor de 
$P$ de 0.0000 , demonstra ser inferior a $1 \%$ e significativo para justificar a relação entre as variáveis.

Averiguando-se o nível de significância, o sinal dos coeficientes e o efeito marginal de cada variável da regressão, constata-se que:

A variável tamanho da firma (tamanho) apresenta-se estatisticamente significativa $(\mathrm{p}=0,004)$ para exercer influência sobre a variável dependente, a um nível de $1 \%$ de significância. O coeficiente positivo $(0,3111)$ demonstra que a variável tamanho faz aumentar para a probabilidade de decisão da firma pela certificação. Assim como o efeito marginal demonstra que o fato da firma possuir um maior tamanho (no caso sendo de médio porte), faz com que a probabilidade de certificação aumente em 5,74\%, tudo mais permanecendo constante.

A este respeito, a literatura destaca que, dentre outros motivos, as empresas menores enfrentarem problemas no acesso ao conhecimento necessário para implementar os procedimentos pertinentes ao processo de certificação (GROLLEAU, MZOUGHI \& PEKOVIC, 2007 apud HUDSON E ORVISKA, 2013), estando neste caso justificado o comportamento positivo da variável com relação à probabilidade de certificação da firma.

No caso da variável localização da firma (local), consta na regressão a probabilidade $(\mathrm{p}=0,023)$, o que representa que ela só tem significância estatística a um nível de $5 \%$ para causar algum efeito na variável dependente. Considerando-se o nível de significância a $5 \%$, cabe analisar o coeficiente da variável local (0,2381), que, tendo sido negativo, demonstra que o impacto do local da firma contribui para a diminuição da propensão à obtenção de certificação, para a amostra em estudo. Com relação ao efeito marginal, pode-se afirmar que, para este grupo de empresas, o fato da firma estar localizada em capitais brasileiras diminui a probabilidade de certificação em 4,39\%, tudo mais permanecendo constante.
Assim sendo, contraria-se a hipótese de que a firma situada na capital tem maior probabilidade de certificação, bem como conflita com o estudo de Hudson e Orviska (2013), que encontram maior probabilidade de empresas com certificação em capitais, onde há mais acesso aos agentes certificadores, bem como os efeitos da certificação podem ser observados mais prontamente.

Analisando a base de dados, percebe-se que dentro da classificação de localização no interior dos Estados constam empresas situadas em regiões metropolitanas ou em regiões com acentuado potencial de desenvolvimento tecnológico. Deste modo, ainda que não estejam alojadas nas capitais dos estados da federação, tais empresas têm também grande probabilidade de certificação.

A variável auditoria externa (audiexterna) apresenta-se estatisticamente significativa $(\mathrm{p}=0,000)$ para exercer impacto sobre a variável dependente, a um nível de $1 \%$ de significância. Além disso, o coeficiente positivo $(0,5349)$ demonstra que a variável audiexterna contribui com o aumento da probabilidade de decisão da firma pela certificação. O efeito marginal demonstra que o fato da firma possuir auditoria externa, torna a probabilidade de certificação maior em 11,95\%, tudo mais permanecendo constante.

É sabido que a auditoria externa é uma das técnicas utilizadas no processo de certificação no Sistema de Gestão da Qualidade, sendo ferramenta imprescindível para a empresa que possui ou deseja implantar a certificação. Assim sendo, o resultado corrobora com a literatura da área (ISO 2009a).

A variável Site Próprio (siteproprio) apresenta significância estatística $(\mathrm{p}=0,000)$ para exercer influência sobre a variável dependente, a um nível de $1 \%$ de significância. O coeficiente positivo $(0,7551)$ demonstra que a variável contribui com o aumento da probabilidade de decisão da firma pela certificação. Pelo cálculo do efeito marginal, afirma-se 
que o fato da firma possuir site próprio, faz com que a probabilidade de certificação aumente em 13\%, tudo mais permanecendo constante.

Com relação ao uso de site próprio para as atividades relacionadas ao negócio, tais como vendas, promoção de produtos, dentre outros, Hudson e Orviska (2013) afirmam que empresas que usam a internet para se conectar com os clientes são muito mais propensas à certificação.

A variável tecnologia estrangeira (tecestrang) apresenta significância estatística $(\mathrm{p}=0,057)$ somente ao nível de $10 \%$. Com relação ao impacto a exercer sobre a variável dependente, o coeficiente positivo $(0,2733)$ demonstra que a contribuição se dá de maneira a aumentar a probabilidade de decisão da firma pela certificação. Assim como o efeito marginal demonstra que o fato da firma utilizar tecnologia estrangeira, faz com que a probabilidade de certificação aumente em 5,68\%, tudo mais permanecendo constante.

$$
\text { A variável Licença de Importação }
$$
(licenseimport) apresenta-se estatisticamente significativa $(\mathrm{p}=0,000)$ para exercer impacto sobre a variável dependente, a um nível de $1 \%$ de significância. Além disso, o coeficiente positivo $(0,4557)$ demonstra que a variável audiexterna contribui com o aumento da probabilidade de decisão da firma pela certificação. De acordo com o efeito marginal, a firma possuir licença de importação faz com que a probabilidade de certificação aumente em 10,08\%, tudo mais permanecendo constante. Esta relação positiva justifica-se dado o acesso que as empresas têm ao mercado exterior, quando da obtenção de uma licença para adquirir, sejam insumos ou componentes, advindos de outros mercados/ países.

\section{CONSIDERAÇÕES FINAIS}

A probabilidade das firmas buscarem uma certificação internacional aumenta quando relacionada ao tamanho da firma, a ocorrência de auditoria externa, a existência de site próprio, ao uso de tecnologia estrangeira e se a empresa recorreu à licença de importação. Entretanto, o estudo aponta, para a amostra de firmas pesquisadas, que a probabilidade de certificação diminui com a localização da firma.

Com base no efeito marginal, as variáveis que mais influenciam o aumento da probabilidade de certificação das pequenas e médias empresas brasileiras, para a amostra em estudo, são: site próprio (13\%), auditoria externa $(11,95 \%)$ e licença de importação $(10,08 \%)$.

\section{REFERÊNCIAS}

ACOSTA, B; PADULA, A.D.; WEGNER, D. Empresas que Possuem Certificações são Mais Inovadoras? Uma Análise no Setor de Produção de Rosas no Equador. SIIMPOI (Anais), 2009.

Disponível em:

<http://www.simpoi.fgvsp.br/arquivo/2009/artigos/E200 9_T00049_PCN24983.pdf〉. Acesso em 15 Mar 2014.

BANCO MUNDIAL (n.d). Enterprise Surveys. Disponível em: 〈www.enterprisesurveys.org〉. Acesso em 17 Abr 2013.

BMI CONSULTORIA (ON LINE). ABNT DIVULGA NÚMERO DE EMPRESAS CERTIFICADAS ISO 9001 NO BRASIL. Disponível em:

$<$ Http://bmiconsultoria.blogspot.Com.Br/2012/12/AbntDivulga-Numero-De-Empresas.Html> Acesso em 02 Abr. 2014.

DEPEXE, M. D.; PALADINI, E. P. Dificuldades Relacionadas à Implantação e Certificação de Sistemas de Gestão da Qualidade em Empresas Construtoras. Revista Gestão Industrial. ISSN 18080448 / v. 03, n. 01: p. 13-25, 2007. Disponível em: <revistas.utfpr.edu.br/pg/index.php/revistagi/article/dow nload/77/74>. Acesso em 20 Mar 2014.

DOSI, G.; PAVITT, K, e SOETE, L.G. The Economics of Technical Change and international Trade, London: Harvester Wheatsheaf, 1990.

GANOTAKIS, Panagiotis; LOVE, James. R\&D, Product Innovation and Exporting: Evidence from UK New Technology Firms. Oxford Economic Papers, vol.63, n.2, April 2011.

GEBREEYESUS, Mulu. Firms' Adoption of International Standards: Evidence from the 
Ethiopian Floriculture Sector. Conference on Economic Development in Africa at the Centre for Studies of African Economies (CSAE), Oxford University, 23rd - 25th March 2014. Disponível em: $<$ https://editorialexpress.com/cgibin/conference/download.cgi?db_name=CSAE2014\&pa per_id=807> Acesso em 30 Mar 2014.

HUDSON, J., \& ORVISKA, M. (2013). Firms' adoption of international standards: One size fits all? Journal of Policy Modeling, Volume 35, Issue 2, March-April 2013, Pages 289-306, ISSN 0161-8938, Disponível em:

<http://dx.doi.org/10.1016/j.jpolmod.2012.04.001> Acesso em 28 Mar 2014.

INMETRO (n.d.). Sistema Brasileiro de Certificação (SBC). Disponível em:

<http://www.inmetro.gov.br/qualidade/comites/sbc.asp> Acesso em 27 Mar 2014.

ISO (2009a). The ISO 9000 family of International Standards. Disponível em: http://www.iso.org/iso/iso_9000_selection_and_use2009.pdf>. Acesso em 31 Mar 2014.

ISO (2009b). The ISO 14000 family of International Standards. Disponível em: 〈http://www.iso.org/iso/theiso14000family_2009.pdf〉. Acesso em 31 Mar 2014.

LA ROVERE, R. L (n.d.). Perspectivas das micro, pequenas e médias empresas no Brasil. Instituto de Economia da UFRJ. Disponível em:

$<$ http://www.ie.ufrj.br/images/pesquisa/publicacoes/rec/ REC\%205/REC_5.Esp_06_Perspectivas_das_micro_pe quenas_e_medias_empresas_no_brasil.pdf $>$. Acesso em 30 Mar 2014.

LEVISTKY, J. Support Systems for SMEs in Developing Countries a Review. Paper commissioned by the Small an Mediym Insustries Branch n.2, Small Medium Programme, UNIDO, 1996.

MOTTA, F.G. Fatores Condicionantes na Adoção de Métodos e Custeio em Pequenas Empresas. Dissertação (Mestrado). São Paulo: USP, 2000.

NASCIMENTO, A.E. Determinantes do Sucesso e Insucesso de Micro e Pequenas Empresas em Floriano - PI (Dissertação de Mestrado). Universidade Federal do Ceará, 2007.

NICOLELLA, G.; MARQUES, J.F.; SKORUPA, L.A. Sistema de Gestão Ambiental: aspectos teóricos e análises de um conjunto de empresas da região de Campinas, SP. Empresa Brasileira de Pesquisa Agropecuária - Embrapa. Documentos 39. São Paulo: Embrapa, 2004. Disponível em: $<$ http://www.cnpma.embrapa.br/download/documentos_ 39.pdf> Acesso em 31 Mar 2014.
OLIVEIRA, M. Implantando a ISO 9001:2000 em Pequenas e Médias Empresas. Salvador: Qualitas, 2001.

PALADINI, E.P. Avaliação Estratégica da Qualidade. São Paulo: Atlas, 2002.

PEKOVIC, S. (2010). The Determinants of ISO 9000 certification: A comparison of the manufacturing and service sectors. Journal of Economic Issues, 44, 895914.

PINO, F. A. Modelos de Decisão Binários: Uma Revisão. Rev. de Economia Agrícola, São Paulo, v. 54, n. 1, p. 43-57, jan./jun. 2007. Disponível em: <ftp://ftp.sp.gov.br/ftpiea/publicacoes/REA0607n4.pdf>. Acesso em 31 Mar 2014.

PUGLIESE, J.B.; SINOARA, R.A.; REZENDE, S.O. Combinação de Regressores Homogêneos e Heterogêneos: Precisão e Compreensibilidade. USP/ Instituto de Ciências Matemáticas e de Computação. Relatório Técnico. São Carlos: Julho, 2013. ISSN 0103-2569. Disponível em:

<http://www.icmc.usp.br/CMS/Arquivos/arquivos_envi ados/BIBLIOTECA_113_RT_203.pdf> Acesso em Acesso em 31 Mar 2014.

RIGIONI, J.R.R.(n.d.a) Quantidade de certificados ISO 9001 no Brasil e no Mundo. Diponível em: <http://www.totalqualidade.com.br/2013/01/quantidadede-certificados-iso-9001-no.html> Acesso em 03 Abr 2014.

RIGIONI, J.R.R.(n.d.b) Quantidade de certificados ISO 14001 no Brasil e no Mundo. Diponível em: <http:/gestaoambiental14001.blogspot.com.br/2013/01/ quantidade-de-certificados-iso-14001-no.html > Acesso em 03 Abr 2014.

ROMANO, C. A.; LIMA , I.A.; LAPOLLI, E.M.; FIALHO, F.A. P. O desafio de apoiar a pequena e média empresa na implantação de programas de qualidade. Congresso Brasileiro de Educação em Engenharia - COBENGE, 2001. Disponível em: <http://www.abenge.org.br/CobengeAnteriores/2001/tra balhos/EQC029.pdf> Acesso em 10 Mar 2014.

SEBRAE - SERVIÇO BRASILEIRO DE APOIO A MICRO E PEQUENA EMPRESA. As Micro e

Pequenas Empresas na Exportação Brasileira. 2011. Disponível em: http://201.2.114.147/bds/bds.nsf/ED8321BE164F5A7C 83257933004D4B5B/\$File/NT00046596.pdf > . Acesso em 01 Mar 2013.

SEBRAE \& ABNT (s.d.). Normas internacionais e MPE - Convênio Sebrae ABNT. Disponível em: <http://portalmpe.abnt.org.br/bibliotecadearquivos/Bibli oteca\%20de\%20Documentos/Normas\%20internacionais $\% 20 \mathrm{e} \% 20 \mathrm{mpe} . \mathrm{pdf}>$ Acesso em 20 Mar 2014. 
SZYSZKA, I. Implantação de Sistemas da Qualidade ISO 9000 e Mudanças Organizacionais. Universidade Federal do Rio Grande do Sul: Porto Alegre, 2001 (Dissertação de Mestrado). Disponível em:

<http://www.lume.ufrgs.br/bitstream/handle/10183/292 5/000328608.pdf?sequence=1 $>$. Acesso em 17 Mar 2014.

WOOLDRIDGE, J. M. Econometric Analysis of Cross Section and Panel Data. Cambrigde: The Mit Press, 1997. 